Supporting information

\title{
Comparative analysis of primer-probe sets for RT-qPCR of COVID-19 causative virus (SARS-CoV-2)
}

\author{
Yujin Jung ${ }^{1, \dagger}$, Gun-Soo Park ${ }^{1,2, \dagger}$, Jun Hye Moon ${ }^{3, \dagger}$, Keunbon $\mathrm{Ku}^{1}$, Seung-Hwa Beak ${ }^{1,4}$, Chang-Seop Lee ${ }^{5,6}$, \\ Seil Kim ${ }^{1,7}$, Edmond Changkyun Park ${ }^{1,8}$, Daeui Park ${ }^{1,4}$, Jong-Hwan Lee ${ }^{1}$, Cheol Woo Byeon ${ }^{3}$, Joong Jin Lee ${ }^{3}$, \\ Jin-Soo Maeng ${ }^{1,2}$, Seong-Jun Kim ${ }^{1}$, Seung Il Kim ${ }^{1,8}$, Bum-Tae Kim ${ }^{1}$, Min Jun Lee ${ }^{3, *}$, and Hong Gi Kim ${ }^{1, *}$ \\ ${ }^{1}$ Center for Convergent Research of Emerging Virus Infection, Korea Research Institute of Chemical \\ Technology, Daejeon 34114, Republic of Korea \\ ${ }^{2}$ Research Group of Food Processing, Korea Food Research Institute, Wanju-gun, Jeollabuk-do 55365, \\ Republic of Korea \\ ${ }^{3}$ Department of Molecular Diagnostics, WELLS BIO, INC, MagokJungang 8-ro 1-gil, Gangseo-gu, Seoul \\ 07795, Republic of Korea
}

${ }^{4}$ Department of Predictive Toxicology, Korea Institute of Toxicology, Daejeon 34114, Republic of Korea

${ }^{5}$ Department of Internal Medicine, Jeonbuk National University Medical School, Jeonju 54986, Republic of Korea

${ }^{6}$ Biomedical Research Institute of Jeonbuk National University Hospital, Jeonju 54907, Republic of Korea

${ }^{7}$ Division of Chemical and Medical Metrology, Center for Bioanalysis, Korea Research Institute of Standards and Science, Daejeon 34113, Republic of Korea

${ }^{8}$ Research Center for Bioconvergence Analysis, Korea Basic Science Institute, Cheongju 28119, Republic of Korea

$\uparrow$ These authors contributed equally to this work.

* Corresponding authors: Hong Gi Kim (tenork@krict.re.kr), and Min Jun Lee (mjlee@wellsbio.net) 


\section{Supporting materials and methods}

\section{Polyacrylamide gel electrophoresis of PCR products}

For electrophoresis of qRT-PCR products, polyacrylamide gel electrophoresis was performed. Briefly, 7.5\% polyacrylamide gel was prepared with 1x TAE buffer (40mM Tris-Acetate, 1mM EDTA, diluted from 50X TAE, Biosesang, South Korea). Total $5 \mu \mathrm{L}$ of each qRT-PCR product mixed with $6 \mathrm{X}$ Loading Dye (Biofact, South Korea) was loaded. The gel was stained in 1x TAE buffer containing 1x RedSafe Nucleic Acid Staining Solution (Intron biotechnology, South Korea) for 30 minutes at ambient temperature. The gel image was captured using ChemiDoc Touch Imaging System (Bio-Rad).

\section{Melting curve analysis}

For the melting curve analysis of qRT-PCR products, the same reagent, instrument, and method are used for qRT-PCR reaction with the following modifications. The reaction solutions were prepared with 1x SYBR Green I dye (Invitrogen) and probes were not added. Melting curves were made from melting steps from each extension temperature to $95^{\circ} \mathrm{C}$.

\section{SARS-CoV-2 genome alignment}

The high-quality and complete SARS-CoV-genome sequenced up to 23 April 2020 retrieved from GISAID ${ }^{1}$. The unique genome sequences were selected from the retrieved sequences using CDHIT [ref: Limin Fu, Beifang Niu, Zhengwei Zhu, Sitao Wu and Weizhong Li, CD-HIT: accelerated for clustering the next generation sequencing data. Bioinformatics, (2012), 28 (23): 3150-3152. doi: 10.1093/bioinformatics/bts565]. The genome sequences with more than one ambiguous sequences were removed using prinseq [ref: Schmieder R and Edwards R: Quality control and preprocessing of metagenomic datasets. Bioinformatics 2011, 27:863-864]. The processed genome sequences were aligned with MAFFT v7.450 [ref: Katoh, Standley 2013 (Molecular Biology and Evolution 30:772-780)] and the mismatchs in primer and probe sites were determined. 


\section{a}

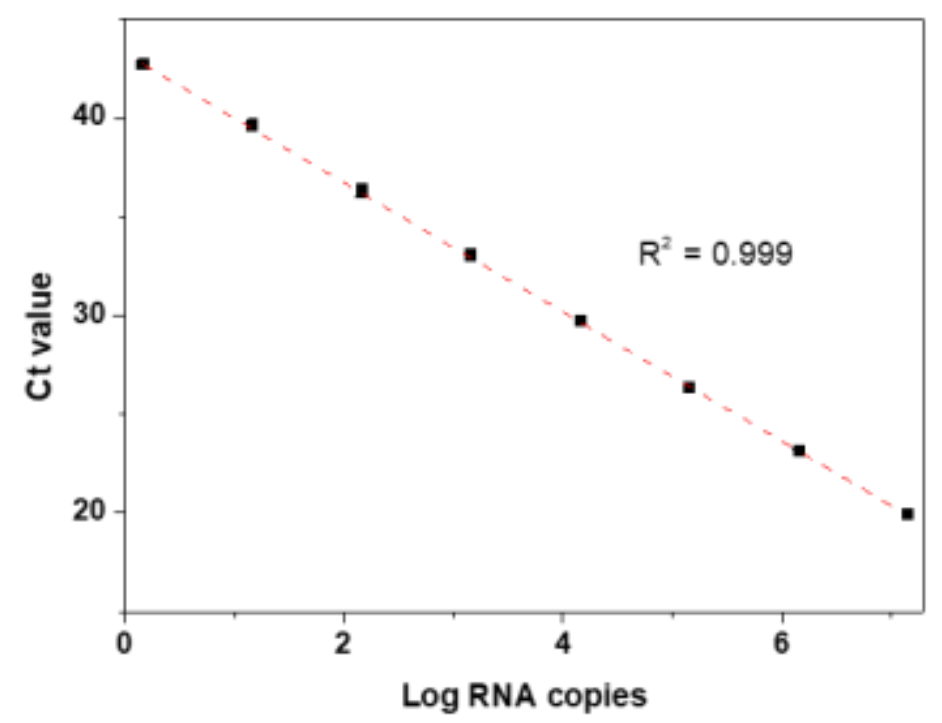

b

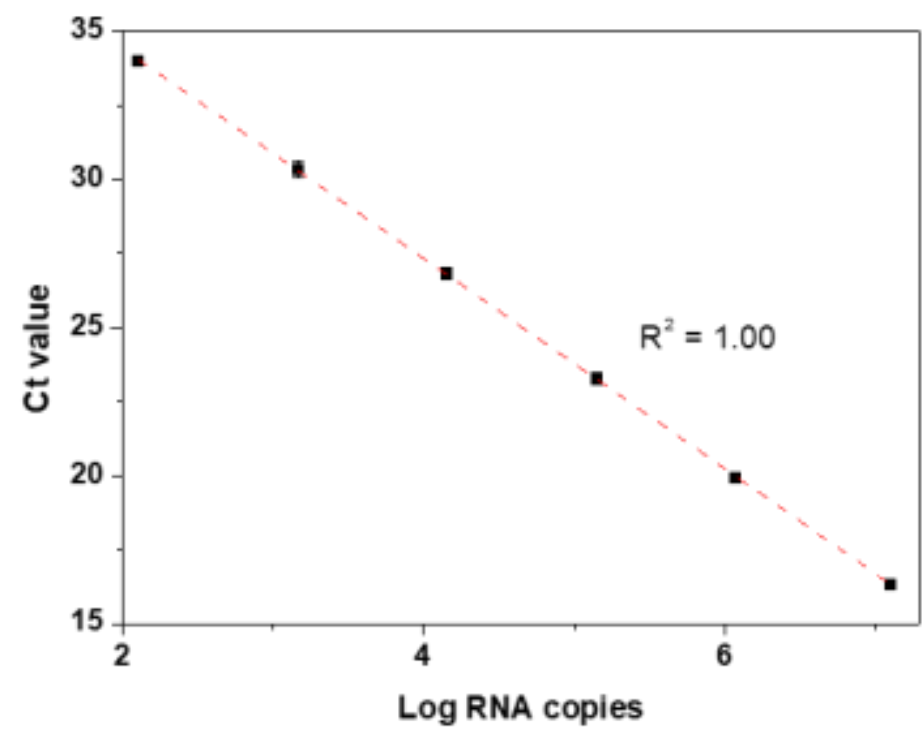

Figure S1. Standard curve analyzed by known amounts of in vitro transcribed RNA standard (E gene) for quantification of (a) the first batch, and (b) the second batch of SARS-CoV-2 RNA. 
Table S1. Comparative analysis of Ct values obtained from a repeated experiment by employing the second batch of SARS-CoV-2 RNA.

\begin{tabular}{|c|c|c|c|c|c|c|c|c|c|c|c|c|c|}
\hline \multirow{2}{*}{$\begin{array}{c}\text { Reaction } \\
\text { temperature }\end{array}$} & \multirow[b]{2}{*}{ Target } & \multirow[b]{2}{*}{ Institute } & \multirow[b]{2}{*}{ Name } & \multicolumn{2}{|c|}{$1.5 \times 10^{4}$ copies } & \multicolumn{2}{|c|}{$1.5 \times 10^{3}$ copies } & \multicolumn{2}{|c|}{$1.5 \times 10^{2}$ copies } & \multicolumn{2}{|c|}{$1.5 \times 10^{1}$ copies } & \multicolumn{2}{|c|}{ NTC } \\
\hline & & & & $\begin{array}{c}\text { Mean } \\
\text { Ct }\end{array}$ & STD & $\begin{array}{c}\text { Mean } \\
\text { Ct }\end{array}$ & STD & $\begin{array}{c}\text { Mean } \\
\text { Ct }\end{array}$ & STD & $\begin{array}{c}\text { Mean } \\
\text { Ct }\end{array}$ & STD & $\begin{array}{c}\text { Mean } \\
\text { Ct }\end{array}$ & STD \\
\hline \multirow{10}{*}{$60^{\circ} \mathrm{C}$} & \multirow{7}{*}{$\mathrm{N}$} & China CDC & $\mathrm{N}$ & 26.50 & 0.072 & 29.80 & 0.070 & 32.40 & 0.118 & 36.09 & 0.149 & 37.53 & 1.529 \\
\hline & & $\mathrm{HKU}$ & HKU-N & 26.88 & 0.044 & 30.21 & 0.062 & 33.32 & 0.079 & 36.47 & 0.183 & $40.03^{*}$ & - \\
\hline & & Japan NIID & NIID_2019-nCOV_N & 24.05 & 0.006 & 27.09 & 0.058 & 30.20 & 0.108 & 33.82 & 0.282 & $\mathrm{~N} / \mathrm{A}$ & $\mathrm{N} / \mathrm{A}$ \\
\hline & & Thailand NIH & WH-NIC N & 29.08 & 0.060 & 32.40 & 0.076 & 34.75 & 0.104 & 38.93 & 1.271 & $44.55^{\star *}$ & $0.141^{\star \star}$ \\
\hline & & \multirow{3}{*}{ US CDC } & 2019-nCoV_N1 & 25.86 & 0.234 & 28.94 & 0.068 & 32.68 & 0.242 & 36.09 & 0.903 & $\mathrm{~N} / \mathrm{A}$ & $\mathrm{N} / \mathrm{A}$ \\
\hline & & & 2019-nCoV_N2 & 24.19 & 0.045 & 27.22 & 0.040 & 30.64 & 0.038 & 34.55 & 0.120 & $\mathrm{~N} / \mathrm{A}$ & $\mathrm{N} / \mathrm{A}$ \\
\hline & & & 2019-nCoV_N3 & 24.22 & 0.030 & 27.40 & 0.072 & 30.60 & 0.095 & 34.00 & 0.557 & $\mathrm{~N} / \mathrm{A}$ & $\mathrm{N} / \mathrm{A}$ \\
\hline & \multirow{3}{*}{ RdRp/Orf1 } & China CDC & ORF1ab & 24.43 & 0.025 & 28.01 & 0.042 & 30.61 & 0.102 & 34.15 & 0.559 & $\mathrm{~N} / \mathrm{A}$ & $\mathrm{N} / \mathrm{A}$ \\
\hline & & Charité & RdRp_SARSr & 28.96 & 0.071 & 32.34 & 0.167 & 35.06 & 0.221 & 37.40 & 1.176 & $42.88^{\star *}$ & $2.291^{* *}$ \\
\hline & & HKU & HKU-ORF1b-nsp14 & 25.53 & 0.127 & 28.91 & 0.055 & 31.74 & 0.062 & 36.14 & 1.293 & $N / A$ & $\mathrm{~N} / \mathrm{A}$ \\
\hline \multirow{10}{*}{$58^{\circ} \mathrm{C}$} & \multirow{7}{*}{$\mathrm{N}$} & China CDC & $\mathrm{N}$ & 27.17 & 0.076 & 30.95 & 0.122 & 33.09 & 0.040 & 37.57 & 0.288 & $36.74^{*}$ & - \\
\hline & & $\mathrm{HKU}$ & HKU-N & 27.78 & 0.114 & 31.95 & 0.047 & 34.10 & 0.165 & 39.18 & 1.308 & $\mathrm{~N} / \mathrm{A}$ & $\mathrm{N} / \mathrm{A}$ \\
\hline & & Japan NIID & NIID_2019-nCOV_N & 25.05 & 0.084 & 28.84 & 0.157 & 31.09 & 0.064 & 34.96 & 0.429 & $\mathrm{~N} / \mathrm{A}$ & $\mathrm{N} / \mathrm{A}$ \\
\hline & & Thailand NIH & WH-NIC N & 29.08 & 0.122 & 33.05 & 0.036 & 35.04 & 0.036 & 39.49 & 1.739 & $40.04^{\star *}$ & $1.414^{* *}$ \\
\hline & & \multirow{3}{*}{ US CDC } & 2019-nCoV_N1 & 26.96 & 0.02 & 30.84 & 0.095 & 32.95 & 0.422 & 36.13 & 1.525 & $N / A$ & $\mathrm{~N} / \mathrm{A}$ \\
\hline & & & 2019-nCoV_N2 & 25.26 & 0.066 & 29.20 & 0.191 & 31.30 & 0.046 & 35.79 & 1.325 & $\mathrm{~N} / \mathrm{A}$ & $\mathrm{N} / \mathrm{A}$ \\
\hline & & & 2019-nCoV_N3 & 25.32 & 0.067 & 29.18 & 0.050 & 31.20 & 0.135 & 35.37 & 0.952 & $\mathrm{~N} / \mathrm{A}$ & $\mathrm{N} / \mathrm{A}$ \\
\hline & \multirow{3}{*}{ RdRp/Orf1 } & China CDC & ORF1ab & 25.26 & 0.060 & 28.98 & 0.137 & 31.39 & 0.348 & 34.76 & 0.635 & $\mathrm{~N} / \mathrm{A}$ & $\mathrm{N} / \mathrm{A}$ \\
\hline & & Charité & RdRp_SARSr & 29.54 & 0.092 & 33.59 & 0.390 & 35.27 & 0.521 & 37.47 & 0.370 & $N / A$ & $\mathrm{~N} / \mathrm{A}$ \\
\hline & & $\mathrm{HKU}$ & HKU-ORF1b-nsp14 & 25.83 & 0.044 & 29.63 & 0.080 & 32.01 & 0.072 & 35.12 & 0.522 & $\mathrm{~N} / \mathrm{A}$ & $N / A$ \\
\hline \multirow{3}{*}{$55^{\circ} \mathrm{C}$} & \multirow{3}{*}{$\mathrm{N}$} & China CDC & $\mathrm{N}$ & 26.3 & 0.182 & 29.67 & 0.307 & 32.92 & 0.231 & 35.63 & 1.081 & $37.68^{* *}$ & $0.622^{* *}$ \\
\hline & & HKU & HKU-N & 25.89 & 0.056 & 29.36 & 0.161 & 32.95 & 0.234 & 37.55 & 0.630 & $38.36^{*}$ & - \\
\hline & & Japan NIID & NIID_2019-nCOV_N & 23.76 & 0.107 & 27.05 & 0.038 & 30.27 & 0.029 & 34.73 & 1.287 & $\mathrm{~N} / \mathrm{A}$ & $\mathrm{N} / \mathrm{A}$ \\
\hline
\end{tabular}




\begin{tabular}{|c|c|c|c|c|c|c|c|c|c|c|c|c|}
\hline & Thailand NIH & WH-NIC N & 27.80 & 0.188 & 31.21 & 0.090 & 34.37 & 0.221 & 36.71 & 0.547 & 38.11 & 0.648 \\
\hline & \multirow{3}{*}{ US CDC } & 2019-nCoV_N1 & 27.15 & 0.069 & 30.88 & 0.312 & 33.13 & 0.350 & 37.41 & 0.366 & $37.54^{*}$ & - \\
\hline & & 2019-nCoV_N2 & 26.38 & 0.012 & 30.97 & 0.093 & 32.5 & 0.137 & 36.37 & 0.100 & $\mathrm{~N} / \mathrm{A}$ & $\mathrm{N} / \mathrm{A}$ \\
\hline & & 2019-nCoV_N3 & 24.78 & 0.615 & 27.53 & 0.042 & 30.55 & 0.095 & 35.83 & 1.421 & $\mathrm{~N} / \mathrm{A}$ & $\mathrm{N} / \mathrm{A}$ \\
\hline & China CDC & ORF1ab & 24.83 & 0.075 & 28.39 & 0.082 & 32.13 & 0.193 & 35.27 & 0.237 & $\mathrm{~N} / \mathrm{A}$ & $\mathrm{N} / \mathrm{A}$ \\
\hline RdRp/Orf1 & Charité & RdRp_SARSr & 29.77 & 0.150 & 32.69 & 0.431 & 37.19 & 0.571 & 37.39 & 1.105 & $\mathrm{~N} / \mathrm{A}$ & $\mathrm{N} / \mathrm{A}$ \\
\hline & HKU & HKU-ORF1b-nsp14 & 25.14 & 0.035 & 28.16 & 0.061 & 31.58 & 0.501 & 34.87 & 0.460 & $\mathrm{~N} / \mathrm{A}$ & $\mathrm{N} / \mathrm{A}$ \\
\hline
\end{tabular}

* The assay showed positive signal from the single reaction of triplicate.

** The assay showed positive signals from the two reactions of triplicate. 

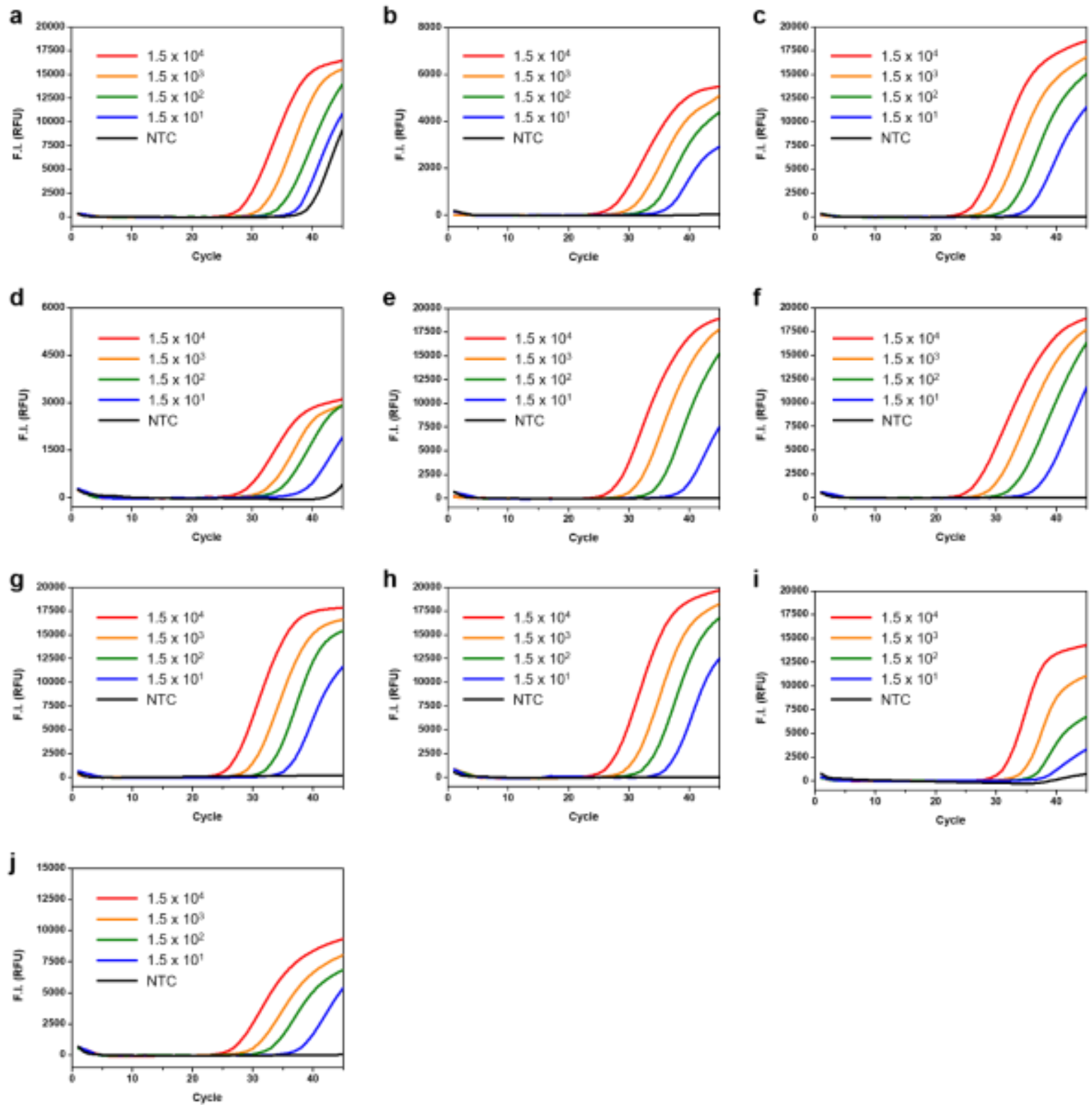

Figure S2. Amplification curves of fluorescence intensity against PCR cycle with the second batch of SARS-CoV-2 RNA (Amplification is performed at 60 $\mathrm{C}$ ). (a) $\mathrm{N}$ (China CDC), (b) HKU-N (HKU), (c) NIID_2019-nCOV_N (Japan NIID), (d) WH-NIC N (Thailand NIH), (e) 2019-nCoV_N1 (US CDC), (f) 2019-nCoV_N2 (US CDC), (g) 2019-nCoV_N3 (US CDC), (h) ORF1ab (China CDC), (i) RdRp_SARSr (Charité), and (j) HKU-ORF1b-nsp14 (HKU) 

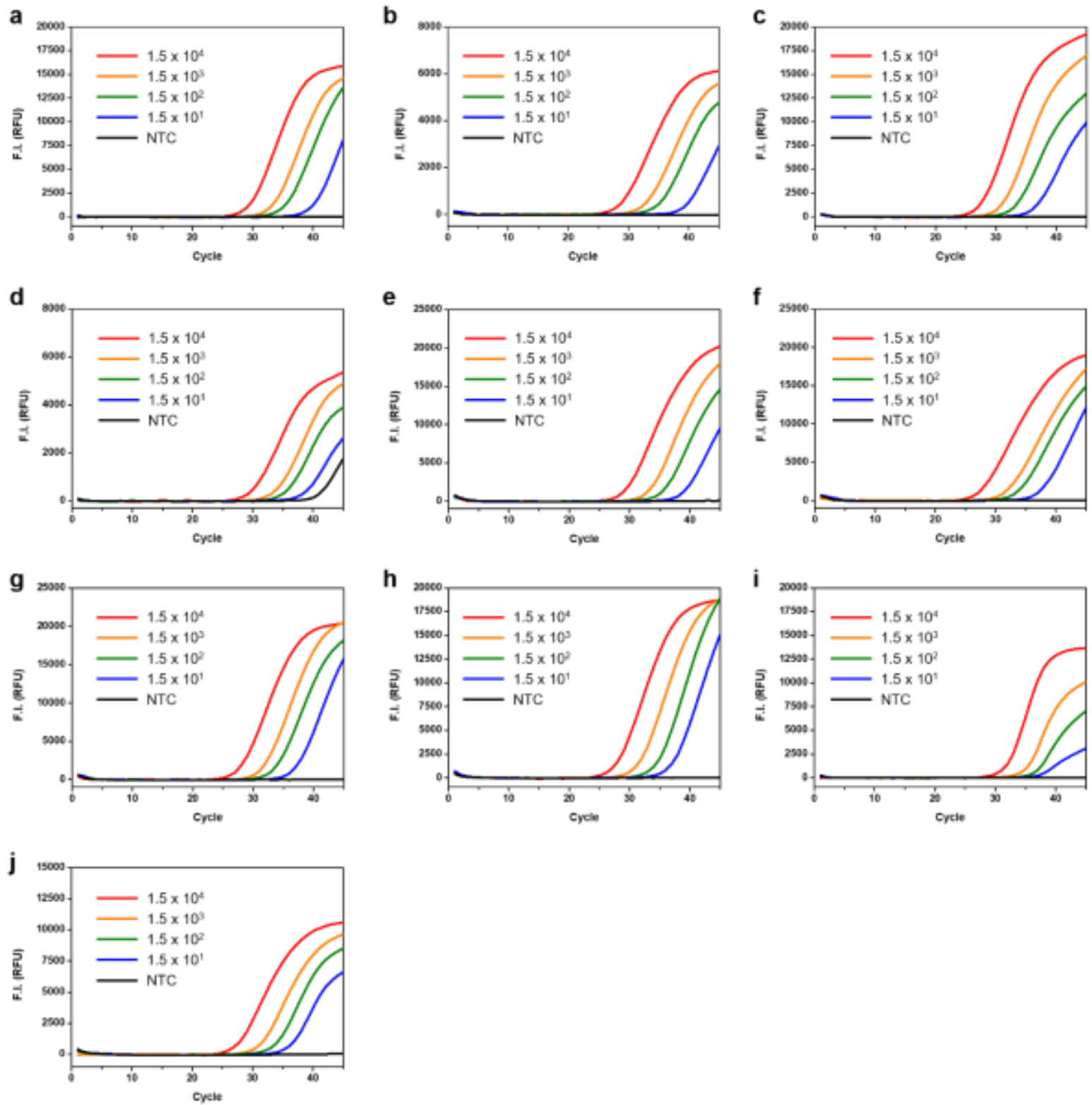

Figure S3. Amplification curves of fluorescence intensity against PCR cycle with the second batch of SARS-CoV-2 RNA (Amplification is performed at $58^{\circ} \mathrm{C}$ ). (a) $\mathrm{N}$ (China CDC), (b) HKU-N (HKU), (c) NIID_2019-nCOV_N (Japan NIID), (d) WH-NIC N (Thailand NIH), (e) 2019-nCoV_N1 (US CDC), (f) 2019-nCoV_N2 (US CDC), (g) 2019-nCoV_N3 (US CDC), (h) ORF1ab (China CDC), (i) RdRp_SARSr (Charité), and (j) HKU-ORF1b-nsp14 (HKU) 

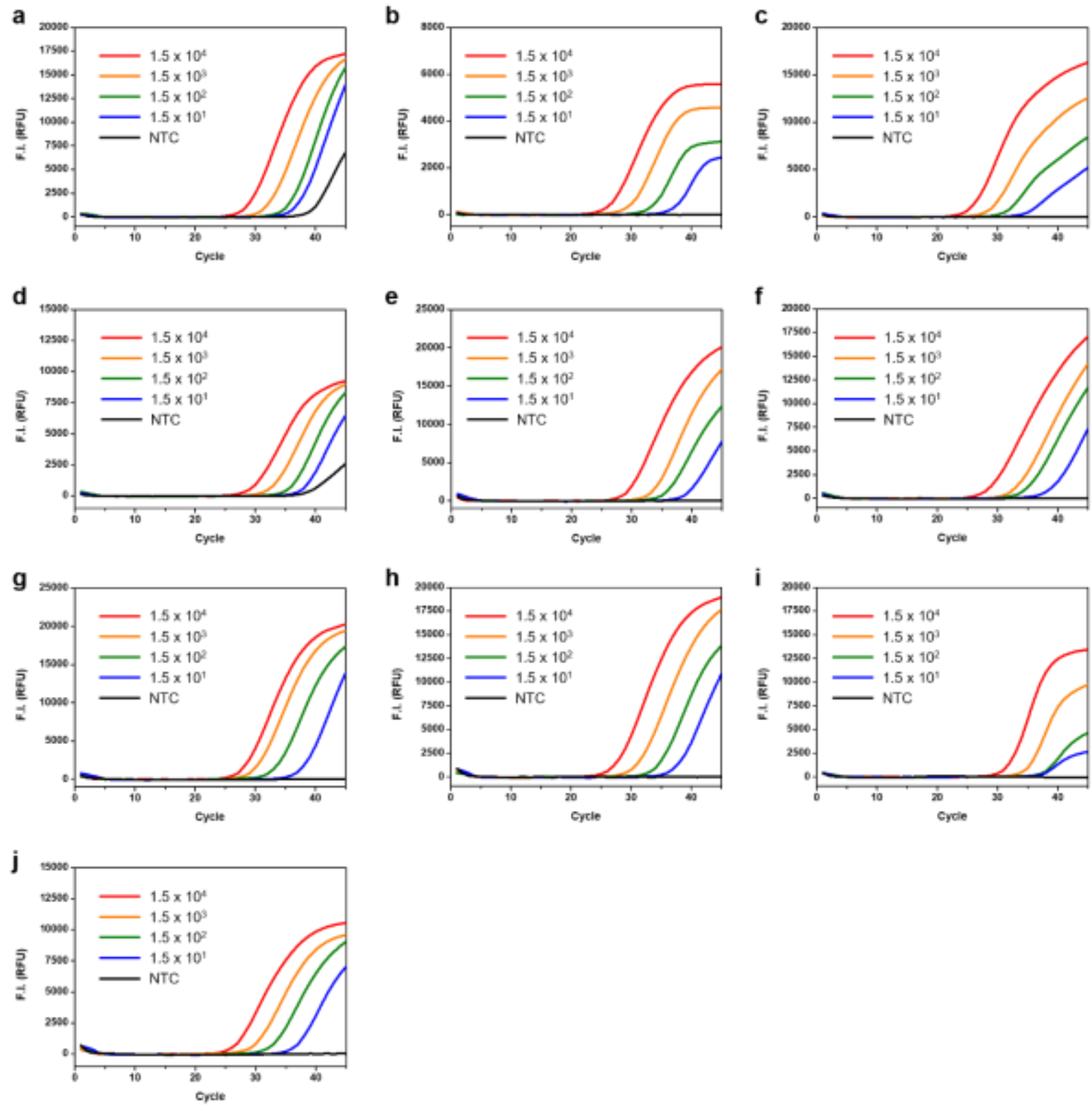

Figure S4. Amplification curves of fluorescence intensity against PCR cycle with the second batch of

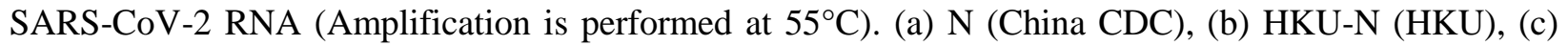
NIID_2019-nCOV_N (Japan NIID), (d) WH-NIC N (Thailand NIH), (e) 2019-nCoV_N1 (US CDC), (f) 2019-nCoV_N2 (US CDC), (g) 2019-nCoV_N3 (US CDC), (h) ORF1ab (China CDC), (i) RdRp_SARSr (Charité), and (j) HKU-ORF1b-nsp14 (HKU) 

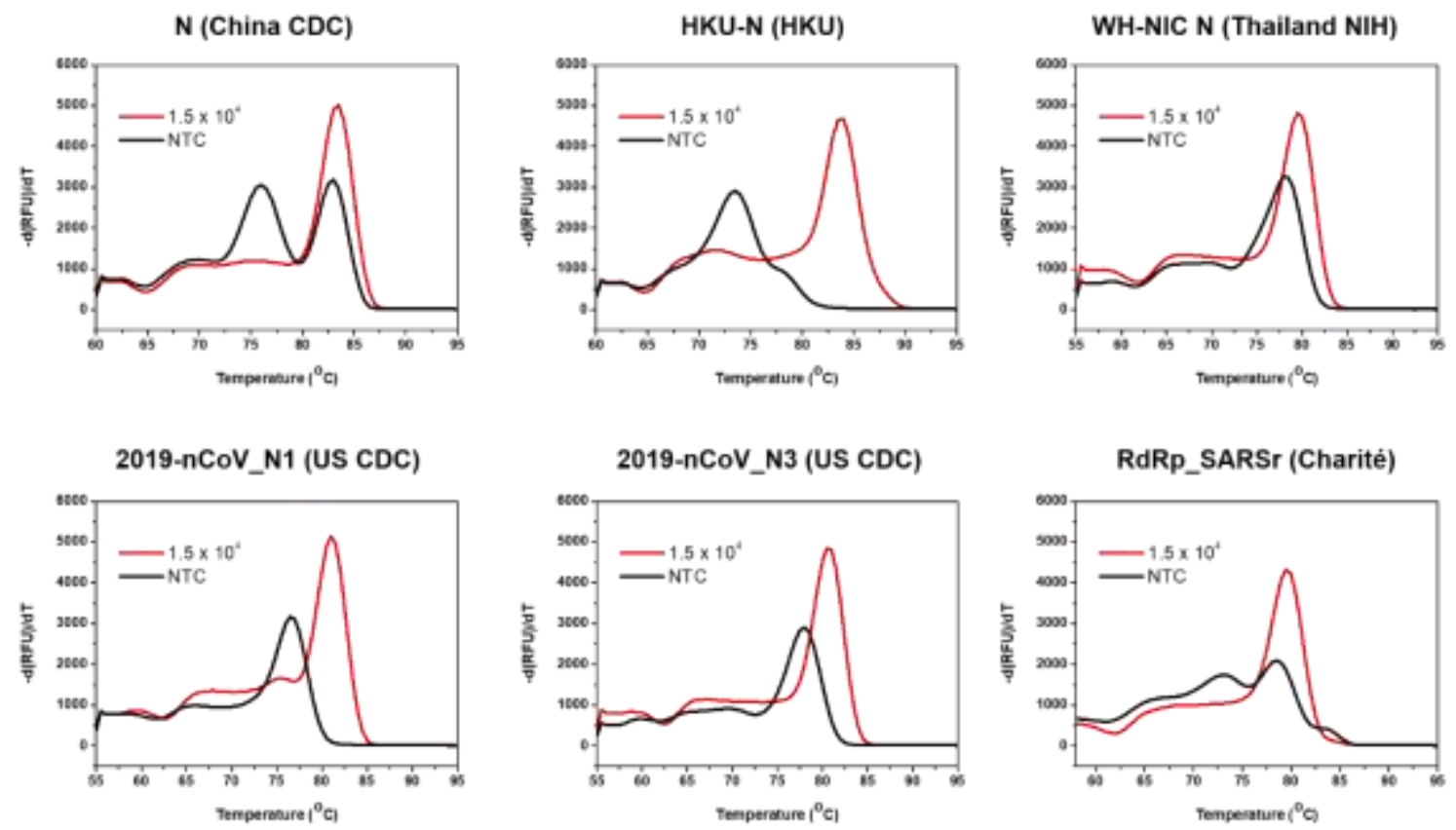

b

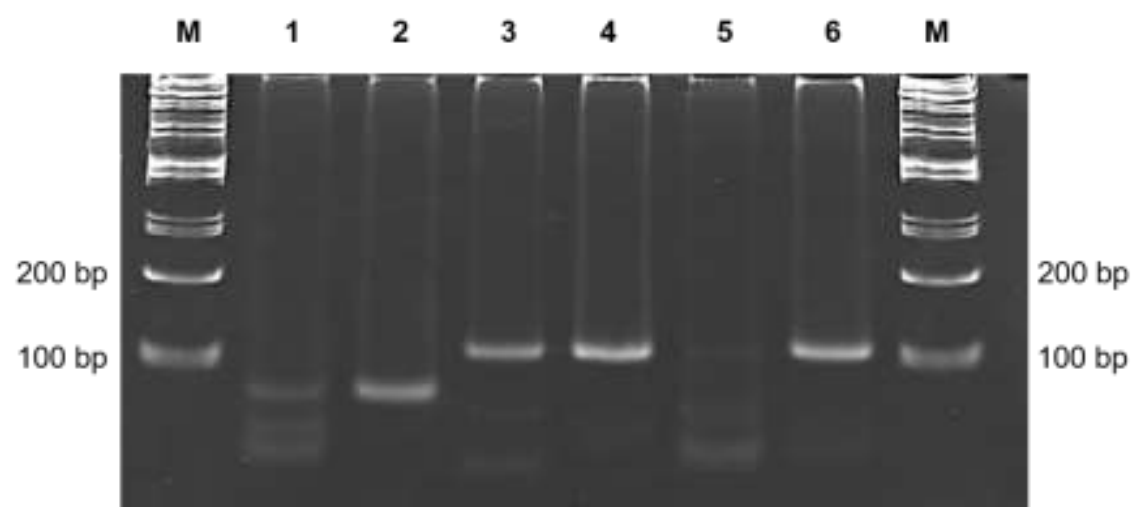

Figure S5. (a) Melting curve analysis and (b) polyacrylamide gel image of PCR products with primerprobe sets that show positive signals in the NTC samples. M : DNA ladder; 1: NTC sample with 2019nCoV_N1 (US CDC); 2: PCR product with 2019-nCoV_N1 (US CDC); 3: NTC sample with N (China CDC); 4: PCR product with N (China CDC); 5: NTC sample with RdRp_SARSr (Charité); 6: PCR product with RdRp_SARSr (Charité) 
Table S2. Clinical characteristics of laboratory-confirmed patients with COVID-19.

\begin{tabular}{|c|c|c|c|c|c|c|}
\hline & Patient 1 (P1) & Patient 2 (P2) & Patient 3 (P3) & Patient 4 (P4) & Patient 5 (P5) & Patient 6 (P6) \\
\hline Age (years)/Sex & $72 / \mathrm{M}$ & $57 / \mathrm{M}$ & $72 / F$ & $38 / \mathrm{M}$ & $21 / F$ & $32 / F$ \\
\hline Fever & - & + & + & - & + & + \\
\hline Chills & - & + & - & - & - & + \\
\hline Cough & + & + & + & - & - & - \\
\hline Dyspnea & - & + & + & - & - & - \\
\hline \multicolumn{7}{|l|}{ Laboratory findings } \\
\hline WBC & 3,570 & 3,710 & 6,720 & 5,810 & 5,800 & 4,280 \\
\hline Hemoglobin & 10.3 & 12.9 & 16.1 & 14 & 14.1 & 11.3 \\
\hline Platelet & 157,000 & 42,000 & 190,000 & 254,000 & 304,000 & 261,000 \\
\hline AST & 23 & 57 & 32 & 39 & 23 & 20 \\
\hline Creatinine & 0.83 & 0.77 & 0.55 & 0.92 & 0.69 & 0.5 \\
\hline \multicolumn{7}{|l|}{ Used drug } \\
\hline Lopinavir/ritonavir & + & + & + & + & + & + \\
\hline Hydroxychloroquine & + & + & + & + & + & + \\
\hline Outcome & Cured & Cured & Cured & Cured & Cured & Cured \\
\hline
\end{tabular}


a

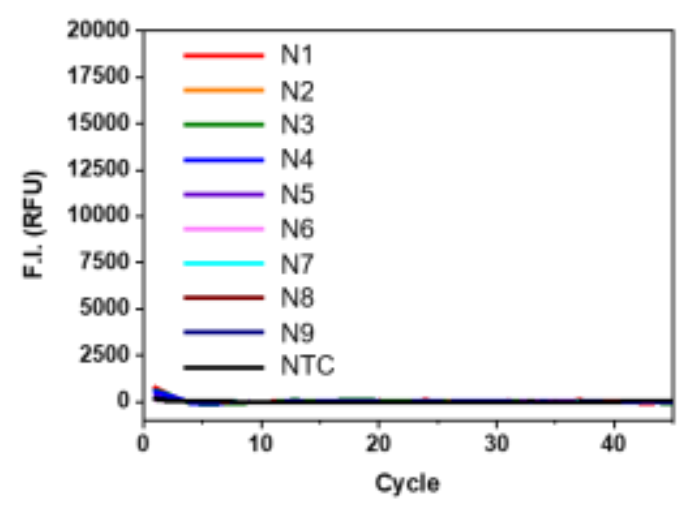

C

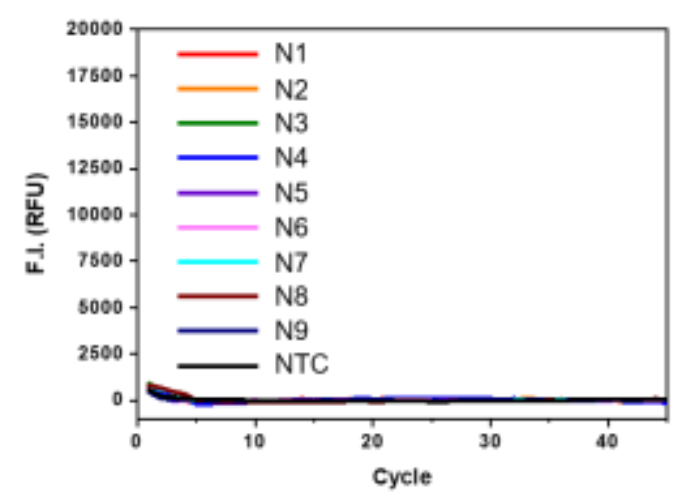

b

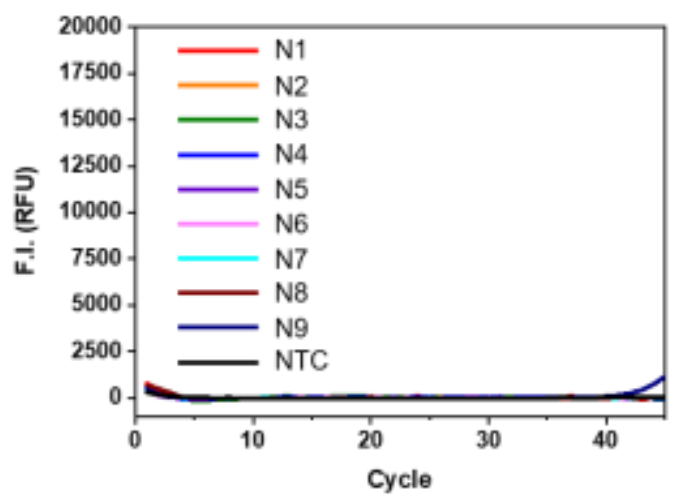

d

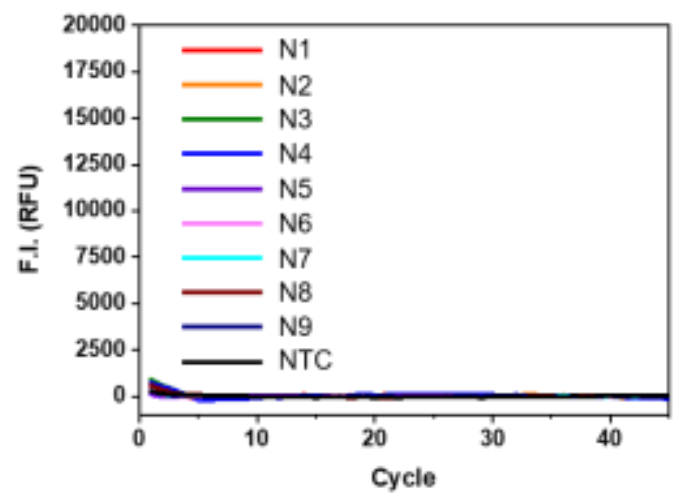

Figure S6. Amplification curves of fluorescence intensity against PCR cycle with the RNAs extracted from clinical samples of healthy subjects. (a) NIID_2019-nCOV_N (Japan NIID), (b) 2019-nCoV_N2 (US CDC), (c) ORF1ab (China CDC), and (d) HKU-ORF1b-nsp14 (HKU) 
a

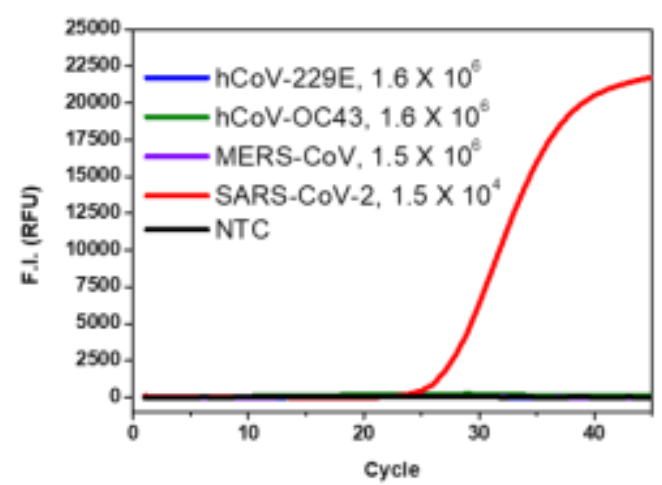

b

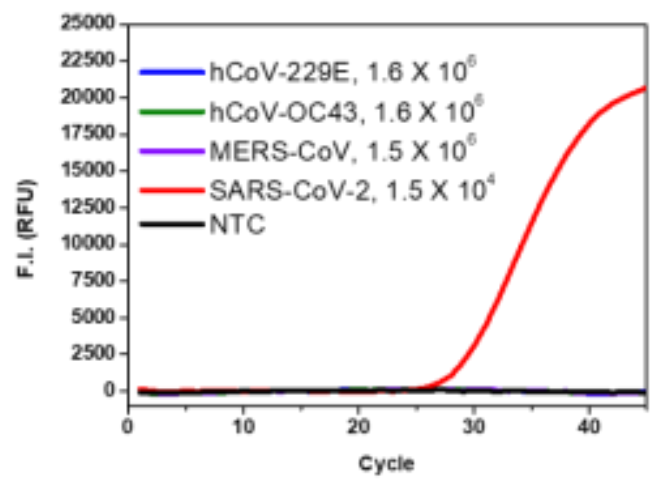

Figure S7. Cross-reactivity test of the RT-qPCR assays for other coronaviruses. (a) NIID_2019-nCOV_N (Japan NIID), (b) ORF1ab (China CDC). 
Table S3. Mismatch nucleotides at the binding sites of the recommended primer-probe sets.

\begin{tabular}{|c|c|c|c|c|c|c|c|c|}
\hline Target & Institute & Name & Type & $\begin{array}{l}\text { Primer/probe position } \\
\qquad\left(5^{\prime} \rightarrow 3^{\prime}\right)\end{array}$ & $\begin{array}{c}\text { Primer/probe } \\
\left.\text { nucleotide ( }{ }^{*} \mathrm{RC}\right)\end{array}$ & $\begin{array}{l}\text { Mismatch } \\
\text { nucleotide }\end{array}$ & $\begin{array}{l}\text { Number of } \\
\text { mismatch }\end{array}$ & Frequency (\%) \\
\hline \multirow{16}{*}{$\mathrm{N}$} & \multirow{16}{*}{$\begin{array}{c}\text { Japan } \\
\text { NIID }\end{array}$} & \multirow{4}{*}{$\begin{array}{l}\text { NIID_2019- } \\
\text { nCOV_N_F2 }\end{array}$} & \multirow{4}{*}{$F$} & 4 & $\mathrm{~T}$ & C & $2 / 3,323$ & 0.06 \\
\hline & & & & 13 & C & $\mathrm{T}$ & $2 / 3,323$ & 0.06 \\
\hline & & & & 16 & $\mathrm{G}$ & $\mathrm{A}, \mathrm{T}$ & $3 / 3,323$ & 0.09 \\
\hline & & & & 20 & $\mathrm{C}$ & $\mathrm{T}$ & $14 / 3,323$ & 0.42 \\
\hline & & \multirow{6}{*}{$\begin{array}{l}\text { NIID_2019- } \\
\text { nCOV_N_R2 }\end{array}$} & \multirow{6}{*}{$\mathrm{R}$} & 4 & $\mathrm{G}^{*}$ & $\mathrm{~T}$ & $1 / 3,323$ & 0.03 \\
\hline & & & & 7 & $C^{*}$ & $\mathrm{~T}$ & $2 / 3,323$ & 0.06 \\
\hline & & & & 10 & $C^{*}$ & $\mathrm{~T}$ & $2 / 3,323$ & 0.06 \\
\hline & & & & 12 & $C^{*}$ & $\mathrm{~T}$ & $1 / 3,323$ & 0.03 \\
\hline & & & & 15 & $C^{*}$ & G & $3,323 / 3,323$ & 100 \\
\hline & & & & 20 & $A^{*}$ & $\mathrm{~T}$ & $1 / 3,323$ & 0.03 \\
\hline & & \multirow{6}{*}{$\begin{array}{l}\text { NIID_2019- } \\
\text { nCOV_N_P2 }\end{array}$} & \multirow{6}{*}{$P$} & 6 & $\mathrm{G}$ & $A, T$ & $2 / 3,323$ & 0.06 \\
\hline & & & & 8 & $\mathrm{G}$ & A & $1 / 3,323$ & 0.03 \\
\hline & & & & 9 & $\mathrm{C}$ & $\mathrm{T}$ & $1 / 3,323$ & 0.03 \\
\hline & & & & 13 & $\mathrm{G}$ & C & $1 / 3,323$ & 0.03 \\
\hline & & & & 17 & $\mathrm{~T}$ & $\mathrm{C}$ & $1 / 3,323$ & 0.03 \\
\hline & & & & 18 & G & $A, T$ & $3 / 3,323$ & 0.09 \\
\hline \multirow{7}{*}{$\begin{array}{l}\operatorname{RdRp} / \\
\text { Orf1 }\end{array}$} & \multirow{7}{*}{$\begin{array}{l}\text { China } \\
\text { CDC }\end{array}$} & \multirow{2}{*}{ ORF1ab-F } & \multirow{2}{*}{$F$} & 4 & $\mathrm{~T}$ & C & $1 / 3,323$ & 0.03 \\
\hline & & & & 17 & C & $\mathrm{T}$ & $1 / 3,323$ & 0.03 \\
\hline & & \multirow{2}{*}{ ORF1ab-R } & \multirow{2}{*}{$\mathrm{R}$} & 9 & $T^{\star}$ & C & $1 / 3,323$ & 0.03 \\
\hline & & & & 15 & $A^{*}$ & $\mathrm{G}$ & $1 / 3,323$ & 0.03 \\
\hline & & \multirow{3}{*}{ ORF1ab-P } & \multirow{3}{*}{$\mathrm{P}$} & 2 & $\mathrm{C}$ & $\mathrm{T}$ & $1 / 3,323$ & 0.03 \\
\hline & & & & 8 & $\mathrm{C}$ & $\mathrm{T}$ & $1 / 3,323$ & 0.03 \\
\hline & & & & 26 & $\mathrm{~T}$ & $\mathrm{G}$ & $51 / 3,323$ & 1.53 \\
\hline
\end{tabular}

${ }^{*} \mathrm{RC}$ : Reverse complement 


\section{References}

1. Shu, Y.; McCauley, J., GISAID: Global initiative on sharing all influenza data-from vision to reality. Eurosurveillance 2017, 22, 30494.

2. Fu, L.; Niu, B.; Zhu, Z.; Wu, S.; Li, W., CD-HIT: accelerated for clustering the next-generation sequencing data. Bioinformatics 2012, 28, 3150-3152.

3. Schmieder, R.; Edwards, R., Quality control and preprocessing of metagenomic datasets. Bioinformatics 2011, 27, 863-864.

4. Katoh, K.; Standley, D. M., MAFFT multiple sequence alignment software version 7: improvements in performance and usability. Molecular biology and evolution 2013, 30, 772-780. 